\title{
Second-order differential superordination for analytic functions in the upper half-plane
}

\author{
Huo Tang ${ }^{a, *}$, H. M. Srivastava ${ }^{b, c}$, Guan-Tie Deng $^{d}$, Shu-Hai Lia \\ a School of Mathematics and Statistics, Chifeng University, Chifeng 024000, Inner Mongolia, People's Republic of China. \\ ${ }^{b}$ Department of Mathematics and Statistics, University of Victoria, Victoria, British Columbia V8W 3R4, Canada. \\ ${ }^{c}$ Department of Medical Research, China Medical University Hospital, China Medical University, Taichung 40402, Taiwan, Republic of \\ China. \\ ${ }^{d}$ School of Mathematical Sciences, Beijing Normal University, Beijing 100875, People's Republic of China.
}

Communicated by M. De La Sen

\begin{abstract}
Let $\Omega$ be a set in the complex plane $\mathbb{C}$. Also let $p(z)$ be analytic in the upper half-plane $\Delta=\{z: z \in \mathbb{C}$ and $\Im(z)>0\}$ and suppose that $\psi: \mathbb{C}^{3} \times \Delta \rightarrow \mathbb{C}$. In this paper, we investigate the problem of determining properties of functions $p(z)$ that satisfy the following second-order differential superordination:

$$
\Omega \subset\left\{\psi\left(\mathrm{p}(z), \mathrm{p}^{\prime}(z), \mathrm{p}^{\prime \prime}(z) ; z\right): z \in \Delta\right\} .
$$

Applications of these results to second-order differential superordination for analytic functions in $\Delta$ are also presented. (C)2017 All rights reserved.

Keywords: Differential subordination, differential superordination, analytic functions, admissible functions, upper half-plane. 2010 MSC: 30C45, 30C80.
\end{abstract}

\section{Introduction}

Let $\Delta$ denote the upper half-plane, that is,

$$
\Delta=\{z: z \in \mathbb{C} \text { and } \mathfrak{I}(z)>0\}
$$

and let $\mathcal{H}[\Delta]$ denote the class of functions $f: \Delta \rightarrow \mathbb{C}$ which are analytic in $\Delta$ and which satisfy the so-called hydrodynamic normalization (see $[1,13,17]$ )

$$
\lim _{\Delta \ni z \rightarrow \infty}[f(z)-z]=0 .
$$

\footnotetext{
${ }^{*}$ Corresponding author

Email addresses: thth2009@163.com (Huo Tang), harimsri@math.uvic.ca (H. M. Srivastava), denggt@bnu .edu.cn (Guan-Tie Deng), lishms66@sina.com (Shu-Hai Li)

doi:10.22436/jnsa.010.10.13
} 
Also let $\mathcal{S}[\Delta]$ denote the class of all functions in $\mathcal{H}[\Delta]$ which are univalent in $\Delta$. Various basic properties concerning functions belonging to the class $\mathcal{S}[\Delta]$ were developed in a series of articles (see, for details, $[10,18,19])$.

A function $f \in \mathcal{H}[\Delta]$, with $f(z) \neq 0$, is said to be starlike in $\Delta$ if and only if

$$
\mathfrak{I}\left(\frac{f^{\prime}(z)}{f(z)}\right)<0 \quad(z \in \Delta) .
$$

We denote by $\mathcal{S}^{*}[\Delta]$ the subclass of $\mathcal{H}[\Delta]$ consisting of functions which are starlike in $\Delta$. We note that, the functions in the class $\mathcal{S}^{*}[\Delta]$ have the property $0 \notin f(\Delta)$.

A function $f \in \mathcal{H}[\Delta]$, with $f(z) \neq z$ and $f^{\prime}(z) \neq 0$, is said to be convex in $\Delta$ if and only if

$$
\Im\left(\frac{f^{\prime \prime}(z)}{f^{\prime}(z)}\right)>0 \quad(z \in \Delta) .
$$

We denote by $\mathcal{K}[\Delta]$ the subclass of $\mathcal{H}[\Delta]$ consisting of functions which are convex in $\Delta$. The classes $\mathcal{S}^{*}[\Delta]$ and $\mathcal{K}[\Delta]$ were introduced by Stankiewicz [17].

We first need to recall the notion of subordination in the upper half-plane.

Let $f$ and $g$ be members of the class $\mathcal{H}[\Delta]$. The function $f$ is said to be subordinate to $g$, or $g$ is said to be superordinate to $f$, if there exists a function $\varphi \in \mathcal{H}[\Delta]$, with $\varphi[\Delta] \subset \Delta$, such that

$$
f(z)=g(\varphi(z)) \quad(z \in \Delta) .
$$

In such a case, we write

$$
f \prec g \quad \text { or } \quad f(z) \prec g(z) \quad(z \in \Delta) .
$$

Furthermore, if the function $g$ is univalent in $\Delta$, then we have the following equivalence ([14]):

$$
f(z) \prec g(z) \quad(z \in \Delta) \Longleftrightarrow f(\Delta) \subset g(\Delta) .
$$

Let $\Omega$ be any set in the complex plane $\mathbb{C}$. Also let $p(z)$ be analytic in $\Delta$ and suppose that $\psi: \mathbb{C}^{3} \times \Delta \rightarrow$ C. Răducanu and Pascu [14] have extended the theory of second-order differential subordination in the unit disk $\mathbb{U}$ to the upper half-plane $\Delta$ by using methods similar to those used in the unit disk introduced by Miller and Mocanu [11]. They determined properties of functions $p(z)$ that satisfy the following second-order differential subordination:

$$
\left\{\psi\left(\mathrm{p}(z), \mathrm{p}^{\prime}(z), \mathrm{p}^{\prime \prime}(z) ; z\right): z \in \Delta\right\} \subset \Omega .
$$

Recently, Tang et al. [20] (see also [22]) have considered the applications of these results to secondorder differential subordination for analytic functions in $\Delta$.

In the following, we will list some definitions and theorem, which are required in our next investigations.

Definition 1.1 ([14]). Let $\psi: \mathbb{C}^{3} \times \Delta \rightarrow \mathbb{C}$ and the function $h(z)$ be univalent in $\Delta$. If the function $p(z)$ is analytic in $\Delta$ and satisfies the following second-order differential subordination:

$$
\psi\left(p(z), p^{\prime}(z), p^{\prime \prime}(z) ; z\right) \prec h(z) \quad(z \in \Delta),
$$

then $p(z)$ is called a solution of the differential subordination. A univalent function $q(z)$ is called a dominant of the solutions of the differential subordination or, more simply, a dominant if $p(z) \prec q(z)$ for all $p(z)$ satisfying Eq. (1.1). A dominant $\widetilde{q}(z)$ that satisfies the following condition:

$$
\widetilde{\mathrm{q}}(z) \prec \mathrm{q}(z) \quad(z \in \Delta)
$$

for all dominants $\mathrm{q}(z)$ of Eq. (1.1) is said to be the best dominant. 
Definition $1.2([11$, p. 403, Definition 8.3i]). Let $\mathcal{Q}(\Delta)$ denote the set of functions $\mathrm{q} \in \mathcal{H}[\Delta]$ that are analytic and injective on $\bar{\Delta} \backslash E(q)$, where

$$
E(q)=\left\{\xi \in \partial \Delta: \lim _{z \rightarrow \xi} q(z)=\infty\right\}
$$

and are such that $\mathrm{q}^{\prime}(\xi) \neq 0$ for $\xi \in \partial \Delta \backslash \mathrm{E}(\mathrm{q})$.

Definition 1.3 ([14]). Let $\Omega$ be a set in $\mathbb{C}$ and $q \in Q(\Delta)$. The class of admissible functions $\Psi_{\Delta}[\Omega, q]$ consists of those functions $\psi: \mathbb{C}^{3} \times \Delta \rightarrow \mathbb{C}$ that satisfy the following admissibility condition:

$$
\psi(\mathrm{r}, \mathrm{s}, \mathrm{t} ; \mathrm{z}) \notin \Omega
$$

whenever

$$
r=q(\xi), \quad s=k q^{\prime}(\xi) \quad \text { and } \quad \Im\left(\frac{t}{q^{\prime}(\xi)}\right) \geqq k^{2} \mathfrak{I}\left(\frac{q^{\prime \prime}(\xi)}{q^{\prime}(\xi)}\right),
$$

where $z \in \Delta, \xi \in \partial \Delta \backslash E(q)$ and $k \geqq 0$.

If $\psi: \mathbb{C}^{2} \times \Delta \rightarrow \mathbb{C}$, then the above admissibility condition reduces to the following form:

$$
\psi\left(\mathrm{q}(\xi), \mathrm{kq}^{\prime}(\xi) ; z\right) \notin \Omega,
$$

where $z \in \Delta, \xi \in \partial \Delta \backslash E(q)$ and $k \geqq 0$.

Theorem 1.4 ([14]). Let $\psi \in \Psi_{\Delta}[\Omega, q]$ and $p \in \mathcal{H}[\Delta]$. If

$$
\psi\left(\mathrm{p}(z), \mathrm{p}^{\prime}(z), \mathrm{p}^{\prime \prime}(z) ; z\right) \in \Omega \quad(z \in \Delta),
$$

then

$$
\mathrm{p}(z) \prec \mathrm{q}(z) \quad(z \in \Delta) .
$$

Following the theory of second-order differential superordinations in the unit disk, which was introduced by Miller and Mocanu [12], Tang et al. [22] considered the dual problem of determining properties of functions $p(z)$ that satisfy the following second-order differential superordination:

$$
\Omega \subset\left\{\psi\left(\mathrm{p}(z), \mathrm{p}^{\prime}(z), \mathrm{p}^{\prime \prime}(z) ; z\right): z \in \Delta\right\} .
$$

In other words, we determine the conditions on $\Omega, \Sigma$, and $\psi$ for which the following implication holds:

$$
\Omega \subset\left\{\psi\left(\mathrm{p}(z), \mathrm{p}^{\prime}(z), \mathrm{p}^{\prime \prime}(z) ; z\right): z \in \Delta\right\} \Longrightarrow \Sigma \subset \mathrm{p}(\Delta),
$$

where $\Sigma$ is any set in $\mathbb{C}$.

If either $\Omega$ or $\Sigma$ is a simply-connected domain, then Eq. (1.2) can be rephrased in terms of superordination. If $p(z)$ is univalent in $\Delta$, and if $\Sigma$ is a simply-connected domain with $\Sigma \neq \mathbb{C}$, then there is a conformal mapping $\mathrm{q}(z)$ of $\Delta$ onto $\Sigma$ such that $\mathrm{q}(0)=\mathrm{p}(0)$. In this case, Eq. (1.2) can be rewritten as follows:

$$
\Omega \subset\left\{\psi\left(\mathrm{p}(z), \mathrm{p}^{\prime}(z), \mathrm{p}^{\prime \prime}(z) ; z\right): z \in \Delta\right\} \Longrightarrow \mathrm{q}(z) \prec \mathrm{p}(z) \quad(z \in \Delta) .
$$

If $\Omega$ is also a simply-connected domain with $\Omega \neq \mathbb{C}$, then there is a conformal mapping $h$ of $\Delta$ onto $\Omega$ such that $h(0)=\psi(p(0), 0,0 ; 0)$. In addition, if the function $\psi\left(p(z), p^{\prime}(z), p^{\prime \prime}(z) ; z\right)$ is univalent in $\Delta$, then Eq. (1.3) can be rewritten as

$$
\mathrm{h}(z) \prec \psi\left(\mathrm{p}(z), \mathrm{p}^{\prime}(z), \mathrm{p}^{\prime \prime}(z) ; z\right) \quad(z \in \Delta) \Longrightarrow \mathrm{q}(z) \prec \mathrm{p}(z) \quad(z \in \Delta) .
$$

There are three key ingredients in the implication relationship Eq. (1.3): the differential operator $\psi$, the set $\Omega$ and the "dominating" function $\mathrm{q}(z)$. If two of these entities were given, one would hope to find conditions on the third entity so that Eq. (1.3) would be satisfied. In this article, we start with a given set $\Omega$ and a given function $\mathrm{q}(z)$, and determine a set of "admissible" operators $\psi$ so that Eq. (1.3) holds true.

We first introduce the following definition. 
Definition 1.5. Let $\psi: \mathbb{C}^{3} \times \Delta \rightarrow \mathbb{C}$ and the function $h(z)$ be analytic in $\Delta$. If the functions $p(z)$ and $\psi\left(p(z), p^{\prime}(z), p^{\prime \prime}(z) ; z\right)$ are univalent in $\Delta$ and satisfy the following second-order differential superordination:

$$
h(z) \prec \psi\left(p(z), p^{\prime}(z), p^{\prime \prime}(z) ; z\right) \quad(z \in \Delta),
$$

then $p(z)$ is called a solution of the differential superordination. An analytic function $q(z)$ is called a subordinant of the solution of the differential superordination or, more simply, a subordinant if $\mathrm{q}(z) \prec$ $p(z)$ for $p(z)$ satisfying Eq. (1.4). A univalent subordinant $\widetilde{q}(z)$ that satisfies the following condition:

$$
\mathrm{q}(z) \prec \widetilde{\mathbf{q}}(z) \quad(z \in \Delta)
$$

for all subordinants $\mathrm{q}(z)$ of Eq. (1.4) is said to be the best subordinant. We note that the best subordinant is unique up to a rotation of $\Delta$.

For $\Omega$ a set in $\mathbb{C}$, with $\psi$ and $p(z)$ as given in Definition 1.5, we suppose that Eq. (1.4) is replaced by

$$
\Omega \subset\left\{\psi\left(\mathrm{p}(z), \mathrm{p}^{\prime}(z), \mathrm{p}^{\prime \prime}(z) ; z\right): z \in \Delta\right\} .
$$

Although this more general situation is a "differential containment", yet we also refer to it as a differential superordination, and the definitions of solution, subordinant and best subordinant as given above can be extended to this more general case.

We will use the following lemma [11, p. 405, Lemma 8.3k] from the theory of second-order differential subordinations in $\Delta$ to determine the subordinants of second-order differential superordinations in $\Delta$.

Lemma 1.6 ([11]). Let $\mathrm{q} \in \mathcal{H}[\Delta]$ and $\mathrm{p} \in \mathcal{Q}(\Delta)$. If $\mathrm{q}(z)$ is not subordinate to $\mathrm{p}(z)$, then there exist points $z_{0}=x_{0}+i y_{0} \in \Delta$ and $\xi_{0} \in \partial \Delta \backslash \mathrm{E}(\mathrm{p})$, and an $\mathrm{m}>0$, such that

(i) $\mathrm{q}\left(z_{0}\right)=\mathrm{p}\left(\xi_{0}\right)$;

(ii) $\mathrm{q}\left(\Delta_{0}\right) \subset \mathrm{p}(\Delta)$, where $\Delta_{\mathrm{y}_{0}}=\left\{z: z \in \mathbb{C}\right.$ and $\left.\mathfrak{I}(z)>\mathrm{y}_{0}\right\}$;

(iii) $\mathrm{q}^{\prime}\left(z_{0}\right)=m p\left(\xi_{0}\right)$;

(iv) $\mathfrak{I}\left(\frac{q^{\prime \prime}\left(z_{0}\right)}{q^{\prime}\left(z_{0}\right)}\right) \geqq m^{2} \Im\left\{\frac{p^{\prime \prime}\left(\xi_{0}\right)}{q^{\prime}\left(z_{0}\right)}\right\}$.

\section{Admissible functions and a fundamental result}

In this section, we first define the class of admissible functions referred to in the preceding section.

Definition 2.1. Let $\Omega$ be a set in $\mathbb{C}$ and $q \in \mathcal{H}[\Delta]$ with $q^{\prime}(z) \neq 0$. The class of admissible functions $\Psi_{\Delta}^{\prime}[\Omega, q]$ consists of those functions $\psi: \mathbb{C}^{3} \times \bar{\Delta} \rightarrow \mathbb{C}$ that satisfy the following admissibility condition:

$$
\psi(r, s, t ; \xi) \in \Omega,
$$

whenever

$$
r=q(z), \quad s=\frac{q^{\prime}(z)}{m} \text { and } \quad \mathfrak{I}\left(\frac{t}{q^{\prime}(z)}\right) \leqq \frac{1}{m^{2}} \mathfrak{I}\left(\frac{q^{\prime \prime}(z)}{q^{\prime}(z)}\right)
$$

where $z \in \Delta, \xi \in \partial \Delta$ and $\mathrm{m}>0$.

If $\psi: \mathbb{C}^{2} \times \bar{\Delta} \rightarrow \mathbb{C}$, then the admissible condition Eq. (2.1) reduces to

$$
\psi\left(\mathrm{q}(z), \frac{\mathrm{q}^{\prime}(z)}{\mathrm{m}} ; \xi\right) \in \Omega \quad(z \in \Delta ; \xi \in \partial \Delta ; \mathrm{m}>0) .
$$

The next theorem is a foundation result in the theory of the second-order differential superordinations in $\Delta$. 
Theorem 2.2. Let $\psi \in \Psi_{\Delta}^{\prime}[\Omega, q]$ and $\mathrm{q} \in \mathcal{H}[\Delta]$. If $\mathrm{p} \in \mathcal{Q}(\Delta)$ and $\psi\left(\mathrm{p}(z), \mathrm{p}^{\prime}(z), \mathrm{p}^{\prime \prime}(z) ; z\right)$ is univalent in $\Delta$, then

$$
\Omega \subset\left\{\psi\left(p(z), p^{\prime}(z), p^{\prime \prime}(z) ; z\right): z \in \Delta\right\}
$$

implies that

$$
\mathrm{q}(z) \prec p(z) \quad(z \in \Delta) .
$$

Proof. Suppose that

$$
\mathrm{q}(z) \nprec \mathrm{p}(z) \quad(z \in \Delta) .
$$

Then, by Lemma 1.6, there exist points $z_{0}=x_{0}+i y_{0} \in \Delta$ and $\xi_{0} \in \partial \Delta \backslash E(p)$, and an $m>0$, that satisfy the conditions (i) to (iv) of Lemma 1.6. Using these conditions with $r=p\left(\xi_{0}\right), s=p^{\prime}\left(\xi_{0}\right), t=p^{\prime \prime}\left(\xi_{0}\right)$, and $\xi=\xi_{0}$ in Definition 2.1, we obtain

$$
\psi\left(p\left(\xi_{0}\right), p^{\prime}\left(\xi_{0}\right), p^{\prime \prime}\left(\xi_{0}\right) ; \xi_{0}\right) \in \Omega,
$$

which contradicts to Eq. (2.2), so we have

$$
\mathrm{q}(z) \prec p(z) \quad(z \in \Delta) .
$$

In the special case, when $\Omega \neq \mathbb{C}$ is a simply-connected domain and $\mathrm{h}$ is a conformal mapping of $\Delta$ onto $\Omega$, we denote this class $\Psi_{\Delta}^{\prime}[\mathrm{h}(\Delta), \mathrm{q}]$ by $\Psi_{\Delta}^{\prime}[\mathrm{h}, \mathrm{q}]$. The following result is an immediate consequence of Theorem 2.2.

Theorem 2.3. Let $\mathrm{q} \in \mathcal{H}[\Delta]$. Also let the function $\mathrm{h}(z)$ be analytic in $\Delta$ and suppose that $\psi \in \Psi_{\Delta}^{\prime}[\mathrm{h}, \mathrm{q}]$. If $\mathrm{p} \in \mathcal{Q}(\Delta)$ and $\psi\left(\mathrm{p}(z), \mathrm{p}^{\prime}(z), \mathrm{p}^{\prime \prime}(z) ; z\right)$ is univalent in $\Delta$, then

$$
h(z) \prec \psi\left(p(z), p^{\prime}(z), p^{\prime \prime}(z) ; z\right) \quad(z \in \Delta)
$$

implies that

$$
\mathrm{q}(z) \prec \mathrm{p}(z) \quad(z \in \Delta) .
$$

Theorems 2.2 and 2.3 can only be used to obtain the subordinants of the differential superordination of the form Eqs. (2.2) or (2.3).

Theorem 2.4. Let the function $h(z)$ be analytic in $\Delta$ and let $\psi: \mathbb{C}^{3} \times \bar{\Delta} \rightarrow \mathbb{C}$. Suppose that the following differential equation:

$$
\psi\left(\mathrm{q}(z), \mathrm{q}^{\prime}(z), \mathrm{q}^{\prime \prime}(z) ; z\right)=\mathrm{h}(z)
$$

has a solution $\mathrm{q} \in Q(\Delta)$. If

$$
\psi \in \Psi_{\Delta}^{\prime}[\mathrm{h}, \mathrm{q}], \quad \mathrm{p} \in \mathcal{Q}(\Delta) \quad \text { and } \quad \psi\left(\mathrm{p}(z), \mathrm{p}^{\prime}(z), \mathrm{p}^{\prime \prime}(z) ; z\right)
$$

is univalent in $\Delta$, then Eq. (2.3) implies that

$$
\mathrm{q}(z) \prec \mathrm{p}(z) \quad(z \in \Delta)
$$

and $\mathrm{q}(z)$ is the best subordinant.

Proof. Since $\psi \in \Psi_{\Delta}^{\prime}[h, q]$, by applying Theorem 2.3, we deduce that $q(z)$ is a subordinant of Eq. (2.3). Since $q(z)$ satisfies Eq. (2.4), it is also a solution of the differential superordination Eq. (2.3). Therefore, all subordinants of Eq. (2.3) will be subordinate to $q(z)$. Hence, $q(z)$ will be the best subordinant of Eq. (2.3).

Next, by making use of the second-order differential superordination results in $\Delta$ obtained in Section 2 (see, for details, Theorems 2.2, 2.3, and 2.4), we determine certain appropriate classes of admissible functions and investigate some second-order differential superordination properties of analytic functions in $\Delta$. It should be remarked in passing that, in recent years, several authors obtained many interesting results associated with second-order and third-order differential subordination and differential superordination in the unit disk. The interested reader may refer to several earlier works including (for example) [2-9, 15, 16, 21, 23, 24]. 


\section{Second-order differential superordinations results}

In this section, we obtain some second-order differential superordination results in $\Delta$. For this aim, the class of admissible functions is given in the following definition.

Definition 3.1. Let $\Omega$ be a set in $\mathbb{C}$ and $q \in \mathcal{H}[\Delta]$ with $\mathrm{q}^{\prime}(z) \neq 0$. The class $\Phi_{\Delta}^{\prime}[\Omega$, $\mathrm{q}]$ of admissible functions consists of those functions $\phi: \mathbb{C}^{3} \times \bar{\Delta} \rightarrow \mathbb{C}$ that satisfy the following admissibility condition:

$$
\phi(u, v, w ; \xi) \in \Omega,
$$

whenever

$$
\mathrm{u}=\mathrm{q}(z), \quad v=\frac{\mathrm{q}^{\prime}(z)}{\mathrm{mq}(z)} \quad(\mathrm{q}(z) \neq 0),
$$

and

$$
\mathfrak{I}\left(\frac{\mathrm{u}\left(w \mathcal{v}+v^{2}\right)}{\mathrm{q}^{\prime}(z)}\right) \leqq \frac{1}{\mathrm{~m}^{2}} \mathfrak{I}\left(\frac{\mathrm{q}^{\prime \prime}(z)}{\mathrm{q}^{\prime}(z)}\right) \quad(z \in \Delta ; \xi \in \partial \Delta ; \mathrm{m}>0) .
$$

Theorem 3.2. Let $\phi \in \Phi_{\Delta}^{\prime}[\Omega, q], f(z) \neq 0$ and $f^{\prime}(z) \neq 0$. If $f \in \mathcal{H}[\Delta]$,

$$
\frac{f^{\prime}(z)}{f(z)} \in Q(\Delta) \quad \text { and } \quad \phi\left(\frac{f^{\prime}(z)}{f(z)}, \frac{f^{\prime \prime}(z)}{f^{\prime}(z)}-\frac{f^{\prime}(z)}{f(z)}, \frac{f(z)\left[f^{\prime}(z) f^{\prime \prime \prime}(z)-\left[f^{\prime \prime}(z)\right]^{2}\right]}{f^{\prime}(z)\left[f(z) f^{\prime \prime}(z)-\left[f^{\prime}(z)\right]^{2}\right]}-\frac{f^{\prime}(z)}{f(z)} ; z\right)
$$

is univalent in $\Delta$, then

$$
\Omega \subset\left\{\phi\left(\frac{f^{\prime}(z)}{f(z)}, \frac{f^{\prime \prime}(z)}{f^{\prime}(z)}-\frac{f^{\prime}(z)}{f(z)}, \frac{f(z)\left[f^{\prime}(z) f^{\prime \prime \prime}(z)-\left[f^{\prime \prime}(z)\right]^{2}\right]}{f^{\prime}(z)\left[f(z) f^{\prime \prime}(z)-\left[f^{\prime}(z)\right]^{2}\right]}-\frac{f^{\prime}(z)}{f(z)} ; z\right): z \in \Delta\right\},
$$

which implies that

$$
q(z) \prec \frac{f^{\prime}(z)}{f(z)} \quad(z \in \Delta) .
$$

Proof. Define the function $\mathrm{p}(z)$ in $\Delta$ by

$$
p(z)=\frac{f^{\prime}(z)}{f(z)} \quad(z \in \Delta) .
$$

A simply calculation yields

$$
\frac{f^{\prime \prime}(z)}{f^{\prime}(z)}-\frac{f^{\prime}(z)}{f(z)}=\frac{p^{\prime}(z)}{p(z)} \quad(z \in \Delta)
$$

Further computations show that

$$
\frac{f(z)\left[f^{\prime}(z) f^{\prime \prime \prime}(z)-\left[f^{\prime \prime}(z)\right]^{2}\right]}{f^{\prime}(z)\left[f(z) f^{\prime \prime}(z)-\left[f^{\prime}(z)\right]^{2}\right]}-\frac{f^{\prime}(z)}{f(z)}=\frac{p^{\prime \prime}(z)}{p^{\prime}(z)}-\frac{p^{\prime}(z)}{p(z)} .
$$

We now define the transformation from $\mathbb{C}^{3}$ to $\mathbb{C}$ by

$$
\mathrm{u}(\mathrm{r}, \mathrm{s}, \mathrm{t})=\mathrm{r}, \quad v(\mathrm{r}, \mathrm{s}, \mathrm{t})=\frac{\mathrm{s}}{\mathrm{r}}, \quad \text { and } \quad w(\mathrm{r}, \mathrm{s}, \mathrm{t})=\frac{\mathrm{rt}-\mathrm{s}^{2}}{\mathrm{rs}} .
$$

Let

$$
\psi(\mathrm{r}, \mathrm{s}, \mathrm{t} ; z)=\phi(\mathrm{u}, v, w ; z)=\phi\left(\mathrm{r}, \frac{\mathrm{s}}{\mathrm{r}}, \frac{\mathrm{rt}-\mathrm{s}^{2}}{\mathrm{rs}} ; z\right)
$$


Using the equations (3.2) to (3.3), we find from Eq. (3.5) that

$$
\psi\left(p(z), p^{\prime}(z), p^{\prime \prime}(z) ; z\right)=\phi\left(\frac{f^{\prime}(z)}{f(z)}, \frac{f^{\prime \prime}(z)}{f^{\prime}(z)}-\frac{f^{\prime}(z)}{f(z)}, \frac{f(z)\left[f^{\prime}(z) f^{\prime \prime \prime}(z)-\left[f^{\prime \prime}(z)\right]^{2}\right]}{f^{\prime}(z)\left[f(z) f^{\prime \prime}(z)-\left[f^{\prime}(z)\right]^{2}\right]}-\frac{f^{\prime}(z)}{f(z)} ; z\right) .
$$

Since $\phi \in \Phi_{\Delta}^{\prime}[\Omega, q]$, Eqs. (3.1) and (3.6) yield

$$
\Omega \subset\left\{\psi\left(\mathrm{p}(z), \mathrm{p}^{\prime}(z), \mathrm{p}^{\prime \prime}(z) ; z\right): z \in \Delta\right\} .
$$

We see from Eq. (3.4) that the admissible condition for $\phi \in \Phi_{\Delta}^{\prime}[\Omega, q]$ in Definition 3.1 is equivalent to the admissible condition for $\psi$ as given in Definition 2.1. Hence $\psi \in \Psi_{\Delta}^{\prime}[\Omega, q]$ and, by Theorem 2.2, we have

$$
\mathrm{q}(z) \prec \mathrm{p}(z) \quad(z \in \Delta)
$$

or, equivalently,

$$
\mathrm{q}(z) \prec \frac{f^{\prime}(z)}{f(z)} \quad(z \in \Delta),
$$

which evidently completes the proof of Theorem 3.2.

If $\Omega \neq \mathbb{C}$ is a simply-connected domain and $\Omega=h(\Delta)$ for some conformal mapping $h(z)$ of $\Delta$ onto $\Omega$, then the class $\Phi_{\Delta}^{\prime}[h(\Delta), \mathrm{q}]$ is written simply as $\Phi_{\Delta}^{\prime}[\mathrm{h}, \mathrm{q}]$. Proceedings similarly as in the preceding section, the following result is an immediate consequence of Theorem 3.2.

Theorem 3.3. Let $\mathrm{q} \in \mathcal{H}[\Delta]$. Also let the function $\mathrm{h}(z)$ be analytic in $\Delta$ and $\phi \in \Phi_{\Delta}^{\prime}[\mathrm{h}, \mathrm{q}]$. If $\mathrm{f} \in \mathcal{H}[\Delta]$, with $\mathrm{f}(z) \neq 0$ and $\mathrm{f}^{\prime}(z) \neq 0$,

$$
\frac{f^{\prime}(z)}{f(z)} \in Q(\Delta) \quad \text { and } \quad \phi\left(\frac{f^{\prime}(z)}{f(z)}, \frac{f^{\prime \prime}(z)}{f^{\prime}(z)}-\frac{f^{\prime}(z)}{f(z)}, \frac{f(z)\left[f^{\prime}(z) f^{\prime \prime \prime}(z)-\left[f^{\prime \prime}(z)\right]^{2}\right]}{f^{\prime}(z)\left[f(z) f^{\prime \prime}(z)-\left[f^{\prime}(z)\right]^{2}\right]}-\frac{f^{\prime}(z)}{f(z)} ; z\right)
$$

is univalent in $\Delta$, then

$$
h(z) \prec \phi\left(\frac{f^{\prime}(z)}{f(z)}, \frac{f^{\prime \prime}(z)}{f^{\prime}(z)}-\frac{f^{\prime}(z)}{f(z)}, \frac{f(z)\left[f^{\prime}(z) f^{\prime \prime \prime}(z)-\left[f^{\prime \prime}(z)\right]^{2}\right]}{f^{\prime}(z)\left[f(z) f^{\prime \prime}(z)-\left[f^{\prime}(z)\right]^{2}\right]}-\frac{f^{\prime}(z)}{f(z)} ; z\right) \quad(z \in \Delta)
$$

implies that

$$
\mathrm{q}(z) \prec \frac{\mathrm{f}^{\prime}(z)}{\mathrm{f}(z)} \quad(z \in \Delta) .
$$

Theorems 3.2 and 3.3 can only be used to obtain subordinations involving the differential superordination of Eqs. (3.1) or (3.7). The following theorem proves the existence of the best subordinant of Eq. (3.7) for a suitably chosen $\phi$.

Theorem 3.4. Let the function $h(z)$ be analytic in $\Delta$ and let $\phi: \mathbb{C}^{3} \times \bar{\Delta} \rightarrow \mathbb{C}$. Suppose that the following differential equation:

$$
\phi\left(\mathrm{q}(z), \frac{\mathrm{q}^{\prime}(z)}{\mathrm{q}(z)}, \frac{\mathrm{q}^{\prime \prime}(z)}{\mathrm{q}^{\prime}(z)}-\frac{\mathrm{q}^{\prime}(z)}{\mathrm{q}(z)} ; z\right)=\mathrm{h}(z)
$$

has a solution $\mathrm{q} \in \mathcal{Q}(\Delta)$. If $\phi \in \Phi_{\Delta}^{\prime}[\mathrm{h}, \mathrm{q}], \mathrm{f} \in \mathcal{H}[\Delta]$, with $\mathrm{f}(z) \neq 0$ and $\mathrm{f}^{\prime}(z) \neq 0$,

$$
\frac{f^{\prime}(z)}{f(z)} \in Q(\Delta) \quad \text { and } \quad \phi\left(\frac{f^{\prime}(z)}{f(z)}, \frac{f^{\prime \prime}(z)}{f^{\prime}(z)}-\frac{f^{\prime}(z)}{f(z)}, \frac{f(z)\left[f^{\prime}(z) f^{\prime \prime \prime}(z)-\left[f^{\prime \prime}(z)\right]^{2}\right]}{f^{\prime}(z)\left[f(z) f^{\prime \prime}(z)-\left[f^{\prime}(z)\right]^{2}\right]}-\frac{f^{\prime}(z)}{f(z)} ; z\right)
$$

is univalent in $\Delta$, then

$$
h(z) \prec \phi\left(\frac{f^{\prime}(z)}{f(z)}, \frac{f^{\prime \prime}(z)}{f^{\prime}(z)}-\frac{f^{\prime}(z)}{f(z)}, \frac{f(z)\left[f^{\prime}(z) f^{\prime \prime \prime}(z)-\left[f^{\prime \prime}(z)\right]^{2}\right]}{f^{\prime}(z)\left[f(z) f^{\prime \prime}(z)-\left[f^{\prime}(z)\right]^{2}\right]}-\frac{f^{\prime}(z)}{f(z)} ; z\right) \quad(z \in \Delta)
$$


implies that

$$
\mathrm{q}(z) \prec \frac{f^{\prime}(z)}{f(z)} \quad(z \in \Delta),
$$

and $\mathrm{q}(z)$ is the best subordinant.

Proof. The proof of Theorem 3.4 is similar to that of Theorem 3.6 in [20] and it is being omitted here.

Definition 3.5. Let $\Omega$ be a set in $\mathbb{C}$ and $\mathrm{q} \in \mathcal{H}[\Delta]$. The class $\Phi_{\Delta, 1}^{\prime}[\Omega, \mathrm{q}]$ of admissible functions consists of those functions $\phi: \mathbb{C}^{2} \times \bar{\Delta} \rightarrow \mathbb{C}$ that satisfy the following admissibility condition:

$$
\phi\left(\mathrm{q}(z), \frac{\mathrm{q}^{\prime}(z)}{\mathrm{m}} ; \xi\right) \in \Omega \quad(z \in \Delta ; \xi \in \partial \Delta ; \mathrm{m}>0) .
$$

Theorem 3.6. Let $\phi \in \Phi_{\Delta, 1}^{\prime}[\Omega, \mathrm{q}]$. If $\mathrm{f} \in \mathcal{H}[\Delta]$, with $\mathrm{f}(z) \neq 0$,

$$
\frac{f^{\prime}(z)}{f(z)} \in \mathcal{Q}(\Delta) \quad \text { and } \quad \phi\left(\frac{f^{\prime}(z)}{f(z)}, \frac{f^{\prime \prime}(z)}{f(z)}-\frac{\left[f^{\prime}(z)\right]^{2}}{[f(z)]^{2}} ; z\right)
$$

is univalent in $\Delta$, then

$$
\Omega \subset\left\{\phi\left(\frac{f^{\prime}(z)}{f(z)}, \frac{f^{\prime \prime}(z)}{f(z)}-\frac{\left[f^{\prime}(z)\right]^{2}}{[f(z)]^{2}} ; z\right): z \in \Delta\right\}
$$

implies that

$$
q(z) \prec \frac{f^{\prime}(z)}{f(z)} \quad(z \in \Delta) .
$$

Proof. Define the function $\mathrm{p}(z)$ in $\Delta$ by

$$
p(z)=\frac{f^{\prime}(z)}{f(z)} \quad(z \in \Delta) .
$$

A simply calculation yields

$$
\frac{f^{\prime \prime}(z)}{f(z)}-\frac{\left[f^{\prime}(z)\right]^{2}}{[f(z)]^{2}}=p^{\prime}(z) .
$$

We next define the transformation from $\mathbb{C}^{2}$ to $\mathbb{C}$ by

$$
u(r, s)=r \quad \text { and } \quad v(r, s)=s .
$$

Then, upon setting

$$
\psi(\mathrm{r}, \mathrm{s} ; z)=\phi(\mathrm{u}, v ; z)=\phi(\mathrm{r}, \mathrm{s} ; z),
$$

the proof will make use of Theorem 1.4. Indeed, if we use the equations (3.9) and (3.10), we find from Eq. (3.12) that

$$
\psi\left(p(z), p^{\prime}(z) ; z\right)=\phi\left(\frac{f^{\prime}(z)}{f(z)}, \frac{f^{\prime \prime}(z)}{f(z)}-\frac{\left[f^{\prime}(z)\right]^{2}}{[f(z)]^{2}} ; z\right)
$$

Since $\phi \in \Phi_{\Delta, 1}^{\prime}[\Omega, q]$, it follows from Eqs. (3.8) and (3.13) that

$$
\Omega \subset\left\{\psi\left(p(z), p^{\prime}(z) ; z\right): z \in \Delta\right\} .
$$


We know from Eq. (3.11) that the admissible condition for $\phi \in \Phi_{\Delta_{1}}^{\prime}[\Omega, q]$ in Definition 3.5 is equivalent to the admissible condition for $\psi$ as given in Definition 2.1. Hence $\psi \in \Psi_{\Delta}^{\prime}[\Omega, q]$ and, by Theorem 2.2, we get

$$
\mathrm{q}(z) \prec \mathrm{p}(z) \quad(z \in \Delta)
$$

or, equivalently,

$$
\mathrm{q}(z) \prec \frac{f^{\prime}(z)}{f(z)} \quad(z \in \Delta) .
$$

In the case $\Omega \neq \mathbb{C}$ is a simply-connected domain with $\Omega=h(\Delta)$ for some conformal mapping $h(z)$ of $\Delta$ onto $\Omega$, the class $\Phi_{\Delta, 1}^{\prime}[h(\Delta), \mathrm{q}]$ is written as $\Phi_{\Delta, 1}^{\prime}[\mathrm{h}, \mathrm{q}]$. Proceedings similarly, the following result is an immediate consequence of Theorem 3.6.

Theorem 3.7. Let $\mathrm{q} \in \mathcal{H}[\Delta]$ and the function $\mathrm{h}(z)$ be analytic in $\Delta$. Also let $\phi \in \Phi_{\Delta, 1}^{\prime}[\mathrm{h}, \mathrm{q}]$. If $\mathrm{f} \in \mathcal{H}[\Delta]$, with $f(z) \neq 0$,

$$
\frac{f^{\prime}(z)}{f(z)} \in \mathcal{Q}(\Delta) \quad \text { and } \quad \phi\left(\frac{f^{\prime}(z)}{f(z)}, \frac{f^{\prime \prime}(z)}{f(z)}-\frac{\left[f^{\prime}(z)\right]^{2}}{[f(z)]^{2}} ; z\right)
$$

is univalent in $\Delta$, then

$$
h(z) \prec \phi\left(\frac{f^{\prime}(z)}{f(z)}, \frac{f^{\prime \prime}(z)}{f(z)}-\frac{\left[f^{\prime}(z)\right]^{2}}{[f(z)]^{2}} ; z\right) \quad(z \in \Delta)
$$

implies that

$$
\mathrm{q}(z) \prec \frac{f^{\prime}(z)}{f(z)} \quad(z \in \Delta)
$$

\section{Acknowledgment}

The research of the first author (Huo Tang) was partly supported by the Natural Science Foundation of the People's Republic of China under Grants 11561001 and 11271045, the Higher School Doctoral Foundation of the People's Republic of China under Grant 20100003110004, the Natural Science Foundation of Inner Mongolia of the People's Republic of China under Grants 2010MS0117, 2014MS0101 and 2017MS0113, the Higher School Foundation of Inner Mongolia of the People's Republic of China under Grants NJZY16251 and NJZY17300 and the Natural Science Foundation of Chifeng of the People's Republic of China.

\section{References}

[1] I. A. Aleksander, V. V. Sobolev, Extremal problems for some classes of univalent functions in the half-plane, Ukr. Math. J., 22 (1970), 291-307. 1

[2] R. M. Ali, V. Ravichandran, N. Seenivasagan, Subordination and superordination of the Liu-Srivastava linear operator on meromorphic functions, Bull. Malays. Math. Sci. Soc., 31 (2008), 193-207. 2

[3] R. M. Ali, V. Ravichandran, N. Seenivasagan, Subordination and superordination on Schwarzian derivatives, J. Inequal. Appl., 2008 (2008), 18 pages.

[4] R. M. Ali, V. Ravichandran, N. Seenivasagan, Differential subordination and superordination of analytic functions defined by the multiplier transformation, Math. Inequal. Appl., 12 (2009), 123-139.

[5] R. M. Ali, V. Ravichandran, N. Seenivasagan, Differential subordination and superordination of analytic functions defined by the Dziok-Srivastava operator, J. Franklin Inst., 347 (2010), 1762-1781.

[6] R. M. Ali, V. Ravichandran, N. Seenivasagan, On subordination and superordination of the multiplier transformation for meromorphic functions, Bull. Malays. Math. Sci. Soc., 33 (2010), 311-324.

[7] M. K. Aouf, T. M. Seoudy, Subordination and superordination of a certain integral operator on meromorphic functions, Comput. Math. Appl., 59 (2010), 3669-3678.

[8] N. E. Cho, O. S. Kwon, S. Owa, H. M. Srivastava, A class of integral operators preserving subordination and superordination for meromorphic functions, Appl. Math. Comput., 193 (2007), 463-474.

[9] N. E. Cho, H. M. Srivastava, A class of nonlinear integral operators preserving subordination and superordination, Integral Transforms Spec. Funct., 18 (2007), 95-107. 2 
[10] G. Dimkov, J. Stankiewicz, Z. Stankiewicz, On a class of starlike functions defined in a half-plane, Annales. Polonici. Math., 55 (1991), 81-86. 1

[11] S. S. Miller, P. T. Mocanu, Differential Subordinations: Theory and Applications, CRC Press, New York, (2000). 1, 1.2, $1,1.6$

[12] S. S. Miller, P. T. Mocanu, Subordinations of differential superordinations, Complex Variables Theory Appl., 48 (2003), 815-826. 1

[13] V. G. Moskvin, T. N. Selakova, V. V. Sobolev, Extremal properties of some classes of conformal self-mapping of the half plane with fixed coefficients, Sibirsk. Mat. Zh., 21 (1980), 139-154. 1

[14] D. Răducanu, N. N. Pascu, Differential subordinations for holomorphic functions in the upper half-plane, Mathematica (Cluj), 36 (1994), 215-217. 1, 1.1, 1.3, 1.4

[15] T. N. Shanmugam, S. Sivasubramanian, H. Srivastava, Differential sandwich theorems for certain subclasses of analytic functions involving multiplier transformations, Integral Transforms Spec. Funct., 17 (2006), 889-899. 2

[16] H. M. Srivastava, D.-G. Yang, N.-E. Xu, Subordinations for multivalent analytic functions associated with the DziokSrivastava operator, Integral Transforms Spec. Funct., 20 (2009), 581-606. 2

[17] J. Stankiewicz, Geometric properties of functons regular in a half-plane, World Sci. Publ., New Jersey, (1992). 1

[18] J. Stankiewicz, Z. Stankiewicz, On the classes of functions regular in a half-plane, Folia Sci. Univ. Tech. Resoviensis Math., 60 (1989), 111-123. 1

[19] J. Stankiewicz, Z. Stankiewicz, On the classes of functions regular in a half-plane, Bull. Polish Acad. Sci. Math., 39 (1991), 49-56. 1

[20] H. Tang, M. K. Aouf, G.-T. Deng, S.-H. Li, Differential subordination results for analytic functions in the upper half-plane, Abstr. Appl. Anal., 2014 (2014), 6 pages. 1, 3

[21] H. Tang, E. Deniz, Third-order differential subordination results for analytic functions involving the generalized Bessel functions, Acta Math. Sci. Ser. B Engl. Ed., 34 (2014), 1707-1719. 2

[22] H. Tang, H. M. Srivastava, G.-T. Deng, Some families of analytic functions in the upper half-plane and their associated differential subordination and differential superordination properties and problems, Appl. Math. Inf. Sci., 11 (2017), 12471257. 1,1

[23] H. Tang, H. M. Srivastava, E. Deniz, S.-H. Li, Third-order differential superordination involving the generalized Bessel functions, Bull. Malays. Math. Sci. Soc., 38 (2015), 1669-1688. 2

[24] H. Tang, H. M. Srivastava, S.-H. Li, L.-N. Ma, Third-order differential subordination and superordination results for meromorphically multivalent functions associated with the Liu-Srivastava operator, Abstr. Appl. Anal., 2014 (2014), 11 pages. 2 